DOI 10.15826/B978-5-7996-3073-7.11

Anna Mazur

University of Economics and Innovation

Lublin, Poland

\title{
The structure of the relationship between physical activity and psychosocial functioning of women and men during the COVID-19 epidemic in Poland
}

Abstract: Since the COVID-19 pandemic is a serious crisis in many countries around the world, it is important to conduct empirical research aimed at identifying risks and factors protecting the functioning of people affected by it $[1,2]$. For this reason, the goal of this research is to determine the structure of the relationship between physical activity and psychosocial functioning of 226 women and 226 men during the COVID-19 epidemic in Poland by looking at connections between physical activity, mental health disorders and cognitive processes and their significance for the quality of social relations. Methodologically, the research relies on IPAQ Questionnaire [3], GHQ-28 Questionnaires [4], TUS Test - 6/9 version [5] and the original SFS Scale. The structural model indicates that physical activity weakens the relationship between mental health disorders and cognitive processes, and gender is the moderator of these relationships. This suggests that physical activity adapted to the condition of health may be an important component of gender-individualized psycho-preventive interventions.

Keywords: physical activity, psychosocial functioning, gender, COVID-19 epidemic

Introduction. The COVID-19 pandemic is a serious health and social problem in many countries around the world. Preliminary empirical evidence suggests that symptoms typical of mental disorders, which are often associated with impaired cognitive functioning, are relatively common psychological responses to this kind of crisis. These types of symptoms may disrupt the social functioning of many people [1]. The literature indicates that regular physical activity may alleviate this risk: by increasing the metabolism of kynurenine and the expression of kynurenine aminotransferase in skeletal muscles, it minimizes the consequences of environ-

(C) Mazur Anna, 2020 
mental stress as well as the symptoms characteristic of neuropsychiatric disorders. Consequently, it improves the quality of social interactions [2]. However, the publications devoted to this issue have appeared only in a few countries affected by the pandemic. As a result, they may not fully reflect the experiences of people living in other parts of the world. Hence, the subject of this research is the structure of the relationship of physical activity and psychosocial functioning of women and men during the COVID-19 epidemic in Poland.

Material and methods. The research was carried out during the COVID-19 epidemic in Poland. The sample consisted of 226 women and 226 men. The following methods were administered: IPAQ Questionnaire [3], GHQ-28 Questionnaires [4], TUS Test - 6/9 version [5] and SFS Scale, created for this research. Statistical calculations were performed with the help of IBM SPSS 25 with AMOS software.

Results. The structural model explains $51.0 \%$ of the variance in the quality of women's and $41.0 \%$ in men's social functioning. Regular physical activity weakens the negative impact of mental health disorders and cognitive processes on the social functioning of our respondents. Moreover, in the population of women the key determinant of the quality of social interactions is the condition of mental health, shaped to a greater extent by cognitive processes related to physical effort than by physical activity alone. On the other hand, the social functioning of men results from the relationship of mental health and the severity of cognitive disorders, moderated by physical effort. In the group of women, physical activity is less important for mental health and cognitive functioning than for men, but mental health is more important for the quality of women's social relationships. In turn, the functioning of cognitive processes determines family, interpersonal and professional interactions of men to a greater extent than of women.

Conclusions. In the estimation of the structural model of the relationship between physical activity and selected aspects of psychosocial functioning, the interactions between physical activity and mental health disorders and cognitive processes were confirmed. Their importance for the quality of social relationships of women and men during the COVID-19 epidemic in Poland was verified. 
- The data indicate that, in the absence of health contraindications, an essential element of interdisciplinary programs supporting psychosocial functioning of adults during an epidemic may be regular physical activity, adjusted to the needs and capabilities of the body.

- The differences between women and men indicate that the psychopreventive interventions should be individualized depending on their gender.

1. Kordovski V.M., Babicz M.A., Ulrich N., Woods S. P. Neurocognitive Correlates of Internet Search Skills for eHealth Fact and Symptom Information in a Young Adult Sample // Percept Mot Skills. 2020. 31512520938178.

2. Su C-H, Chuang H-C, Hong C-J. Physical exercise prevents mice from L-Kynurenine-induced depression-like behavior // Asian J Psychiatry. 2020. 48:101894.

3. Biernat E., Stupnicki R., Gajewski A. K. Physical exercise prevents mice from L-Kynurenine-induced depression-like behavior // Polish version. Wych Fiz Sport. 2007. № 51(1). P. 47-54.

4. Makowska Z., Merecz D. Polish adaptation of David Goldberg's General Health Questionnaire GHQ-12 and GHQ-28. Łódź: Instytut Medycyny Pracy, 2001. [in Polish].

5. Ciechanowicz A., Stańczak J. Attention and Perceptiveness Tests 6/9 Version — standards for adults. Warsaw: Psychological Test Laboratory, 2017. 